Documentation et bibliothèques

DOCUMENTATION BIBLIOTHËUES

Nature et rôle d'un réseau

Nature and function of a network

Esencia y papel de una red de bibliotecas

\title{
Guy Cloutier
}

Volume 28, numéro 3, juillet-septembre 1982

URI : https://id.erudit.org/iderudit/1053729ar

DOI : https://doi.org/10.7202/1053729ar

Aller au sommaire du numéro

\section{Éditeur(s)}

Association pour l'avancement des sciences et des techniques de la documentation (ASTED)

ISSN

0315-2340 (imprimé)

2291-8949 (numérique)

Découvrir la revue

Citer cet article

Cloutier, G. (1982). Nature et rôle d'un réseau. Documentation et bibliothèques, 28(3), 101-103. https://doi.org/10.7202/1053729ar

\section{Résumé de l'article}

Les nouvelles technologies de traitement et de diffusion de l'information sont à l'origine du concept de réseau automatisé de bibliothèques. Un réseau permet de réaliser collectivement des objectifs qui dépassent les capacités individuelles de chaque participant. Il engendre une redéfinition du rôle des bibliothèques, qui deviennent des pourvoyeuses d'information. Mais les réseaux ne sont que des moyens. Ils ont plusieurs visages. Leur forme et leur efficacité sont subordonnées à la définition par les composantes de leurs besoins et de leurs objectifs.
Tous droits réservés @ $@$ Association pour l'avancement des sciences et des techniques de la documentation (ASTED), 1982
Ce document est protégé par la loi sur le droit d'auteur. L’utilisation des services d'Érudit (y compris la reproduction) est assujettie à sa politique d'utilisation que vous pouvez consulter en ligne.

https://apropos.erudit.org/fr/usagers/politique-dutilisation/ 


\title{
Nature et rôle d'un réseau
}

\author{
Guy Cloutier* \\ Université de Sherbrooke \\ Sherbrooke
}

Les nouvelles technologies de traitement et de diffusion de l'information sont à l'origine du concept de réseau automatisé de bibliothèques. Un réseau permet de réaliser collectivement des objectifs qui dépassent les capacités individuelles de chaque participant. II engendre une redéfinition du rôle des bibliothèques, qui deviennent des pourvoyeuses d'information. Mais les réseaux ne sont que des moyens. Ils ont plusieurs visages. Leur forme et leur efficacitè sont subordonnées à la définition par les composantes de leurs besoins et de leurs objectifs.

\section{Nature and function of a network}

New technologies for processing and distributing information are at the root of the concept of an automated library network. Through a network, objectives can be collectively attained which transcend individual capabilities. This brings forth a rethinking of the role of the libraries which become information purveyors. Nevertheless, networks are merely a means. They offer many facets. Their structure as well as their effectiveness are conditioned by the definition of their needs and objectives as laid by the members.

\section{Esencia y papel de una red de bibliotecas}

Las nuevas técnicas del tratamiento de datos y de la difusión de la información son el origen del concepto de la automatización en redes de bibliotecas. Una red permite la realización colectiva de los objetivos que sobrepasan las capacidades individuales de cada participante. Genera una redefinición del papel de las bibliotecas que se convierten en proveedores de información. Por otra parte, las redes no son más que medios con muchas facetas. Sus formas y su eficacia estan subordinadas a la definición por las componentes de sus necesidades y de sus objetivos.

Dans le contexte de la révolution informatique qui progresse à un rythme exponentiel, il est de mise que les intervenants que sont les spécialistes de l'information et de la documentation s'interrogent sur le rôle qu'ils accordent et accorderont aux nouvelles technologies de traitement et de diffusion de l'information.

C'est à la suite des développements technologiques des dernières années qu'on en est arrivé au concept de réseau automatisé de bibliothèques, afin de permettre à celles-ci de réaliser deux objectifs importants: le partage des ressources, et la réalisation de projets coopératifs reliés dans un premier temps au traitement de la documentation.

C'est grâce entre autres au leadership et à la clairvoyance de la Bibliothèque nationale du

\footnotetext{
* L'auteur est directeur du Service des bibliothèques.
}

Canada que la discussion s'est amorcée au début des années soixante-dix; c'est également grâce à l'initiative du Service de la bibliothèque de l'Université de Toronto qu'est né et s'est développé UTLAS (University of Toronto Library Automation Systems) à laquelle entreprise furent associées la majorité des bibliothèques universitaires du Québec et certaines bibliothèques spécialisées. Plus près de nous, l'Université du Québec innova en développant le réseau BADADUQ (Banque de données à accès direct de l'Université du Québec) axé essentiellement sur le repérage de l'information, contrairement à UTLAS qui était et demeure encore un projet collectif de traitement informatisé de la documentation. Tout récemment, I'Université du Québec à Montréal (UQAM) développait un système intégré de gestion de bibliothèques pouvant fonctionner en réseau, SIGIRD (Système intégré de gestion informatisée des ressources documentaires). 
De plus, en mars 1981, deux comités furent créés par le Conseil consultatif de la Bibliothèque nationale du Canada afin de le conseiller sur différentes questions relatives au développement d'un réseau de bibliothèques décentralisé. Le Comité du réseau de services bibliographiques et de communications fera des recommandations sur divers aspects reliés à la création et au fonctionnement d'un réseau de communication bibliographique, alors que le Comité du réseau de ressources présentera des recommandations sur toute question touchant le partage des ressources documentaires au pays.

Un réseau informatique de bibliothèques permet de réaliser collectivement, grâce aux techniques d'informatique et de communication, des objectifs qu'aucun des participants ne pourrait réaliser individuellement. II présuppose que les buts et objectifs des participants sont similaires en ce qui concerne la dimension ressource, la dimension bibliographique ainsi que la dimension communication des relations qui caractérisent les échanges entre les membres du réseau.

La dimension ressource implique que le réseau est constitué de façon à garantir la disponibilité et l'accès à la documentation susceptible d'être demandée par un des participants, notamment par la coordination et la rationalisation du développement des collections, pour s'assurer que la documentation requise sera rassemblée et accessible à tous.

La dimension bibliographique recouvre la création et l'utilisation des bases de données bibliographiques requises pour assurer l'exécution des différentes fonctions bibliographiques telles que le catalogage, la localisation, etc.

La dimension communication du réseau se rapporte à toute l'infrastructure de télécommunication nécessaire au transfert des données et, éventuellement, au transfert des documents euxmêmes et ce, pour permettre tant l'exécution des fonctions bibliographiques que l'accès aux ressources du réseau.

S'il est une question qui a engendré beaucoup de discussions durant les dernières années dans le milieu des bibliothèques, c'est bien toute cette question des réseaux informatisés de bibliothèques, dont l'implantation et le développement sont la suite logique des efforts entrepris précédemment pour l'automatisation des bibliothèques; et déjà pointe à l'horizon une nouvelle phase ou étape, consécutive également aux deux premières, soit l'interconnexion des réseaux.

Il est important de souligner que toute cette évolution traduit un changement important dans la mission traditionnelle de nos bibliothèques. Alors que la mission première de la bibliothèque a toujours été d'être une pourvoyeuse de documentation imprimée et audiovisuelle, il devient de plus en plus évident - sans doute davantage au niveau des bibliothèques de recherche et des bibliothèques spécialisées - que la bibliothèque devient en premier lieu une pourvoyeuse d'intormation, suite entre autres à l'explosion de la documentation, à l'automatisation et à l'apparition des bases de données. L'accès à l'information prend de plus en plus le pas sur l'accès à la documentation comme mission première; l'usager dans sa quête d'information, fichiers, bases de données, bibliographies, etc., identifie un certain nombre de documents disponibles soit localement soit à l'intérieur d'un réseau quelconque. Or cet accès tant à l'information qu'à la documentation n'est possible en terme de délais raisonnables que par l'intermédiaire des réseaux informatisés; cet accès, il sera éventuellement élargi grâce à l'interconnexion des réseaux et accéléré par les futures technologies de transmission de texte.

Les développements en matière de réseaux informatisés de bibliothèques sont, au-delà des avantages économiques et autres qu'ils représentent, une nécessité dans toute socièté le moindrement organisée. II est en effet impératif d'offrir un accès rapide et structuré aux masses d'information générées à un rythme effarant; il est tout aussi impératif par la suite de livrer rapidement la marchandise, en l'occurence le contenu ou le document lui-même. La seule solution de remplacement serait l'autosuffisance institutionnelle, ce qui est évidemment impensable.

II ne faut jamais perdre de vue que les ordinateurs et toute la technologie des communications ne sont que des outils et que c'est à nous d'abord de déterminer à quelles fins ces nouvelles technologies seront utilisées dans nos bibliothèques.

Si nous n'avons pas fait l'effort de préciser nos objectifs, en ce qui est des besoins à satisfaire chez nos usagers, nous nous réveillerons avec une "quincaillerie" qui nous donnera peut-être l'air d'être à la mode, mais qui engendrera beaucoup de déceptions et de frustrations parce qu'elle nous entraînera à sacrifier une partie de nos objectifs institutionnels, ou parce qu'elle drainera une trop grande partie de nos ressources compte tenu des avantages obtenus.

Il va de soi que les besoins d'une grande bibliothèque universitaire ou d'une bibliothèque de recherche varient énormément comparativement à ceux d'une bibliothèque publique ou d'une bibliothèque scolaire et qu'en conséquence les composantes bibliographiques et lẹs eléments de communication ne peuvent être complètement similaires; les objectifs, le volume d'activités et les ressources sont trop différents.

On ne saurait encore une fois trop insister sur le fait que les nouvelles technologies ne sont qu'un moyen de mieux servir nos usagers, et sur la nécessité que nous définissions nous-mêmes nos propres objectifs afin de bien adapter l'outil à la satisfaction des besoins de nos usagers.

C'est cette même définition de nos besoins et de nos objectifs - exercice qui n'a jamais été fait au Québec - qui pourra nous éclairer sur les orientations à prendre en matière de développement de 
réseaux informatiques de bibliothèques. Par exemple, est-il préférable d'avoir en premier lieu un réseau régional, regroupant les différentes bibliothèques d'une même région quitte, par la suite, à développer des réseaux sur une base institutionnelle e.g. réseau des bibliothèques de l'Estrie et réseau des bibliothèques universitaires du Québec? Un second modèle pourrait être un ensemble de réseaux sur une base institutionnelle, e.g. le réseau des bibliothèques universitaires, le réseau des bibliothèques de collège, etc... Un troisième modèle - le moins souhaitable pour des raisons de flexibilité, d'objectifs institutionnels, de besoins, de normes etc. - pourrait être un réseau unique regroupant toutes les bibliothèques quels que soient leurs objectifs, leur rattachement institutionnel, leur dimension ou leur localisation.
Il y a de fait plusieurs possibilités. qu'il nous appartient de définir en fonction de nos objectifs. priorités et ressources; les spécialistes de l'informatique et des communications sauront très bien adapter ou développer l'outil qui satisfera aux exigences du modèle que nous aurons défini.

Nous devons prendre conscience du rôle qui est le nôtre face au développement et à l'implantation de réseaux informatiques de bibliothèques. II nous appartient de représenter les intérêts de nos usagers et de nous assurer que nos objectifs institutionnels seront bien servis lorsque le moment sera venu de faire le saut.

La meilleure façon de nous préparer, c'est sans aucun doute d'accumuler le maximum d'information et de nous familiariser le plus possible avec ces nouveaux concepts.

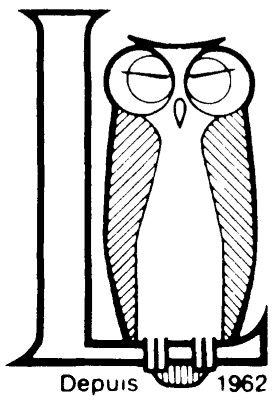

\section{R.M. LEDUC \& CIE BIBLIOTHEQUES}

\section{MAINTENANT : SERVICE DE RÉPARATION ET RELIURE DE LIVRES ET PÉRIODIQUES.}

PROTECTEURS DE LIVRES EN FILM POLYESTER • PELLICULES AUTO-ADHÉSIVES • RUBAN DE LECTO-CONTACT OU PELLIMAT • DÉVIDOIRS • TRICOL • PROTÉGE-FICHES VERTICAL, HORIZONTAL OU SURELEVÉ - FICHESGUIDE - PROTĖGE-PÉRIODIQUES - POCHETTES DE VOLUME - ÉTIQUETTES COLLANTES LECTO-DURO ÉTIQUETTES AUTO-ADHÉSIVES • COLLE PLAXANOL • PERFORATEURS • MATÉRIEL D'EXPÉDITION • RUBANS AUTO-ADHÉSIFS • DOSSIERS SUSPENDUS • CHEMISES - ENVELOPPES PAPIER KRAFT REMBOURRÉ.

\section{C.P. 1379, Marieville, Qué. JOL 1J0 (514) 658-0661}

